\title{
The effect of dentifrice including dental type silica, tocopherol acetate, sodium fluoride and sodium pyrophosphate on mineral density in enamel
}

\author{
Ho-Ki Jung ${ }^{1}$, Sun-Young Chung ${ }^{1}$, Yong-Su Ahn ${ }^{1}$, Kyong-Hoon Shin ${ }^{2}$, Ja-Won Cho ${ }^{1}$ \\ ${ }^{1}$ Department of Preventive Dentistry, College of Dentistry, Dankook University, Cheonan, Republic of Korea \\ ${ }^{2}$ Aekyung Industrial Co., Ltd. R\&D Division Dental Care Team, Daejeon, Republic of Korea
}

\begin{abstract}
Objectives: In this study, we aimed to investigate the preventive and protective effects of new dentifrice containing dental type silica, tocopheryl acetate, fluorides, and sodium pyrophosphate on the mineral density of teeth and demineralization of tooth surfaces.

Methods: A total of 119 bovine teeth pre-treated with the new dentifrice at three different concentrations for the experiment were randomly allocated into two control (DW and PW) and one experimental (EC) groups. The enamel surfaces of all bovine teeth were demineralized using an artificial demineralization solution. The dentifrice was diluted with distilled water (DW) at 1:1, 1:2, and 1:3 ratios. The samples were treated with the demineralization solution for $4 \mathrm{~h}$ after treatment with the supernatants of each diluted dentifrice for $30 \mathrm{~min}$, and this procedure was repeated 3 times over a period of 24 hours. The samples were examined using micro-CT to determine the amount of reduced bone mineral density (BMD) comparing the control and experimental dentifrices. The surface changes of the samples were also examined using the scanning electron microscope (SEM).

Results: The average BMD of the bovine enamel surface between the treated and non-treated area with the dimineralization solution was significantly different in the control, DW, PW 1:1, PW 1:2, and PW 1:3 groups. However, there was no significant difference observed in the experimental groups, including EC 1:1, EC 1:2, and EC 1:3. The average BMD of the dimineralized surfaces based on the results of the 7 groups was significantly higher in every EC group when compared to the DW and three PW groups. Conclusions: The new dentifrice containing dental type silica, tocopheryl acetate, fluorides, and sodium pyrophosphate is effective in inhibiting the decrease in BMD and demineralization of enamel surface, which was observed when the new dentifrice and demineralization solution was repeatedly applied to the samples for 24 hours.
\end{abstract}

Key Words: Dentifrices, Sodium fluoride, Sodium pyrophosphate

Copyright (C) 2021. Korean Academy of Preventive Dentistry. All rights reserved.

This is an Open Access article distributed under the terms of the Creative Commons Attribution Non-Commercial License (http://creativecommons.org/licenses/ by-nc/4.0) which permits unrestricted non-commercial use, distribution, and reproduction in any medium, provided the original work is properly cited. 